\title{
Detection of multidrug-resistant Salmonella enterica subsp. enterica serovar Typhi isolated from Iraqi subjects
}

\author{
Hamzah Abdulrahman Salman' ${ }^{10}$, Ali Mohammed Abdulmohsen² ${ }^{(i)}$, Mays Noori Falih² (iD) and Zahraa Mohmoud Romi² \\ 1. Department of Medical Laboratory Techniques, College of Medical Sciences Techniques, The University of Mashreq, \\ Baghdad, Iraq; 2. The Biological Society of Iraq, Baghdad, Iraq. \\ Corresponding author: Hamzah Abdulrahman Salman, e-mail: hamza.alayash@gmail.com \\ Co-authors: AMA: alibio1982@gmail.com, MNF: maysbio2003@gmail.com, ZMR: zahraa.alromi@gmail.com \\ Received: 06-03-2021, Accepted: 18-06-2021, Published online: 26-07-2021
}

doi: www.doi.org/10.14202/vetworld.2021.1922-1928 How to cite this article: Salman HA, Abdulmohsen AM, Falih MN, Romi ZM (2021) Detection of multidrug-resistant Salmonella enterica subsp. enterica serovar Typhi isolated from Iraqi subjects, Veterinary World, 14(7): 1922-1928.

\begin{abstract}
Background and Aim: Enteric fever initiated by Salmonella enterica subsp. enterica serovar Typhi (S. Typhi) is among the most consistent disease worldwide, particularly in developing countries. The present study aimed to isolate and identify $S$. Typhi from typhoid suspected patients and determine their antibacterial susceptibility testing.

Materials and Methods: Thirty blood samples were collected from typhoid suspected patients in Baghdad, Iraq. The samples were cultured on SS agar and XLD agar for screening of $S$. Typhi. The suspected colonies were picked up and subjected to Vitek 2 compact for biochemical identification and antibacterial susceptibility testing of the organisms. Molecular identification of the isolates was performed by real-time polymerase chain reaction (RT-PCR).

Results: Black colonies were observed on cultured plates. Out of 30 samples, 27 and 29 isolates were identified as $S$. Typhi using Vitek 2 compact and RT-PCR, respectively. The data of the present study revealed that the strains of $S$. Typhi were showing multidrug resistance. All $S$. Typhi strains exhibited resistance to penicillins (ticarcillin and piperacillin), cephalosporins $4^{\text {th }} \mathrm{G}$ (cefepime), and monobactam (aztreonam). However, all the strains showed susceptibility against carbapenems (imipenem and meropenem) and tetracycline (minocycline).
\end{abstract}

Conclusion: RT-PCR and Vitek 2 compact showed a high level of accuracy in the detection of $S$. Typhi. Multidrug resistance was observed, which is an alert for the reduction of antibiotic consumption.

Keywords: antibiotics susceptibility, enteric fever, multidrug-resistant, Salmonella, typhoid fever, Vitek 2 compact.

\section{Introduction}

Enteric fever is a severe infectious disease caused by the human-restricted agent Salmonella enterica subsp. enterica. The genus Salmonella has over 2500 serotypes classified into two distinct species: S. enterica and Salmonella bongori. S. enterica subsp. enterica consists of 1500 serotypes [1]. S. enterica subsp. enterica serovar Typhi ( $S$. Typhi) and Salmonella Paratyphi A, B, and C are extremely adapted to the human host, acting as their typical reservoir [2].

The transport of the disease is either waterborne, foodborne, or through significant person-to-person contact [3]. Salmonella is one of the world's most common causes of food poisoning. Such bacteria can infect a wide range of food products such as poultry, dairy products, or meal, including those of animal origin [4]. Salmonella can also cause diseases in the farming of poultry and pigs [5]. The economic loss caused by an infected diet is one of the main concerns for Salmonella [6]. In Asia and Africa, the disease

Copyright: Salman, et al. Open Access. This article is distributed under the terms of the Creative Commons Attribution 4.0 International License (http://creativecommons.org/licenses/ by/4.0/), which permits unrestricted use, distribution, and reproduction in any medium, provided you give appropriate credit to the original author(s) and the source, provide a link to the Creative Commons license, and indicate if changes were made. The Creative Commons Public Domain Dedication waiver (http:// creativecommons.org/publicdomain/zero/1.0/) applies to the data made available in this article, unless otherwise stated. continues to be extremely severe, with close to 21 million cases and an estimated 220,000 deaths each year. Enteric fever in endemic areas, especially resource limited, is a significant public health concern [3]. An epidemiological study about Iraq stating that thousands of people are dying every year due to the deficiency of fresh water and contamination of rivers by sewage and factories [7].

The high rate of occurrence of typhoid fever was worsened by the rise of antibiotic-resistant $S$. enterica subsp. enterica. To minimize the spread of resistant strains, the Centers for Disease Control and Prevention classified drug-resistant $S$. Typhi as a critical problem that needs regular monitoring and prevention [8]. Antibiotic-resistant $S$. Typhi is the greatest challenge worldwide, especially in countries with poor sanitation, medication facilities, and low income [9]. Emerging multidrug resistance has led to an increment in the death rate as antibiotics are no longer effective. A very distressing investigation about multidrug-resistant (MDR) $S$. Typhi in the Middle East and Central Asia reports that Iraq has the highest prevalence of MDR $S$. Typhi followed by Pakistan [10].

The standard technique to identify Salmonella, widely applied for 60 years, includes detecting 3 -antigenic locations (somatic $\mathrm{O}$ and two antigenic flagella $\mathrm{H}$ ) using a particular antiserum by slide agglutination, the Kauffmann-White-Le Minor scheme. Despite 
its global use, this technology is time-intensive, not consistently effective, and needs skilled personnel [11]. It is challenging, particularly in children with uncommon symptoms, to distinguish enteric fever from other undifferentiated febrile diseases, including influenza, leptospirosis, dengue, or malaria $[12,13]$. Widal test is widely used in many endemic countries, although the sensitivity and specificities are poorly defined as the most common diagnostic procedures [12,14]. Tools such as polymerase chain reaction (PCR) have become central in detecting and typing contagious diseases by providing rapidness, sensitivity, and specificity $[3,15,16]$, however, it is unaffected whether patients were consumed antibiotics or not.

It is very essential to have a rapid and accurate detection for Salmonella spp. However, traditional techniques such as the Widal test, Enzyme-linked immunosorbent assay, culturing, and biochemical tests are still regularly used to detect Salmonella spp. However, these techniques are laborious and inaccurate. In third world countries, such as Iraq, the rate of detection of bacteria causing enteric fever as well as their antibiotics resistance is hard to assess because of lack of monitoring, insufficient reporting, and publication. For the above reasons, the study aimed to isolate and identify $S$. Typhi from typhoid suspected patients and determine their antibacterial susceptibility pattern.

\section{Materials and Methods}

\section{Ethical approval and Informed consent}

Ethical approval was not needed for this study. We have obtained the samples from the Al-Nokhba Diagnostic Centre, collected by trained personnel and with the doctor's supervision. The objective of the study was described to all the patients in detail, and a signed consent form was collected.

\section{Study period and location}

The samples were collected from January 2020 to June 2020 in Baghdad, Iraq. The samples were processed in the Hematology Unit and Microbiology Unit at Al-Nokhba Diagnostic Centre.

\section{Bacterial isolates}

The study enrolled patients with high fever $\left(>38^{\circ} \mathrm{C}\right)$ who approached Al-Nokhba Diagnostic Centre in Baghdad, Iraq. Thirty blood samples were collected from suspected typhoid patients suffering from fever, headache, and stomach pain. All the patients did not receive antibiotics for at least 2 weeks before sample collection. Blood samples were cultured on Salmonella Shigella (SS) agar (HiMedia, India), and Xylose Lysine Deoxycholate (XLD) agar (HiMedia, India). The selected colonies were subjected to further characterization.

\section{Biochemical tests}

All the suspected colonies were biochemically tested by Vitek 2 compact (bioMérieux, France) using Vitek 2 GN card according to the manufacturer's instructions. Three to five fresh colonies were transferred into two tubes containing $3 \mathrm{~mL}$ of normal saline. The suspension was inoculated into the Vitek 2 compact with a Gram-negative identification test. The biochemical tests employed in the present study are mentioned in Table-1.

\section{DNA extraction}

The DNAs of the isolates were extracted using Wizard genomic DNA purification kit (Promega, USA) as instructed by the manufacturer. One hundred microliters of DNA from each sample were eluted and stored at $-20^{\circ} \mathrm{C}$ until use for molecular detection.

\section{Real-time PCR (RT-PCR) assays}

Salmonella enterica subsp. enterica was detected by targeting invasion A (InvA) gene using genesig ${ }^{\circledR}$ Standard Kit (Primerdesign Ltd., UK). All RT-PCR assays were performed according to the manufacturer's protocol. Positive and negative controls were involved. The positive control was provided with the same kit above, while RNase-/DNase-free water was used as a negative control. All the cycles were performed on StepOnePlus RT-PCR System (Thermo Fisher Scientific, Massachusetts, US).

\section{Antibiotics susceptibility pattern}

The isolates were tested for their susceptibility to antibiotics by Vitek 2 compact (bioMérieux, France) using GN cassette AST-N222 card (bioMérieux, France) according to the manufacturer's instructions. The turbidity was adjusted to 0.5 as per McFarland standard by DensiCHEK Plus. The suspension was inoculated into the Vitek 2 compact with GN cassettes AST card. According to Vitek 2 compact system special software, interpretation of the results was performed as explained by the manufacturer's instructions. Following the Clinical Laboratory Standards Institute criteria, the susceptibility data were analyzed [17]. The antibiotics employed in this study were ticarcillin, ticarcillin/clavulanic acid, piperacillin, cefepime, ceftazidime, aztreonam, amikacin, gentamicin, tobramycin, ciprofloxacin, trimethoprim/ sulfamethoxazole, minocycline, piperacillin/tazobactam, imipenem, and meropenem.

\section{Results}

Thirty blood samples acquired from 30 suspected typhoid patients were involved in this study. The colony of Salmonella was shown as black in color on SS agar and XLD agar due to the production of $\mathrm{H}_{2} \mathrm{~S}$ (Figure-1). Of the sample size, 27 isolates were recognized as $S$. Typhi based on biochemical tests (Table-1). In contrast, 29 isolates were identified as $S$. Typhi using RT-PCR (Figure-2).

The antibiotics susceptibility pattern of $S$. Typhi isolates is demonstrated in Table-2. Among the tested antibiotics, the strains of $S$. Typhi shown resistance to 12 antibiotics belong to seven classes. Of them, all $S$. Typhi strains were resistant to penicillins (ticarcillin and piperacillin), cephalosporins $4^{\text {th }} \mathrm{G}$ (cefepime), and 
Table-1: The representative of biochemical characterization of 27 strains of Salmonella Typhi by Vitek 2 compact GN card.

\begin{tabular}{|c|c|c|c|c|c|}
\hline No. & Biochemical test & Results & No. & Biochemical tests & Results \\
\hline 1. & ala-phe-pro-arylamidase & - & 25. & D-glucose & + \\
\hline 2. & $\mathrm{H} 2 \mathrm{~S}$ production & - & 26. & D-mannose & + \\
\hline 3. & Beta-glucosidase & - & 27. & Tyrosine arylamidase & - \\
\hline 4. & L-proline arylamidase & - & 28. & Citrate(sodium) & - \\
\hline 5. & Saccharose/sucrose & - & 29. & Beta-n-acetyl-galactosaminidase & - \\
\hline 6. & L-Lactate alkalinization & + & 30. & L-Histidine assimilation & - \\
\hline 7. & Glycine arylamidase & - & 31. & Ellman & - \\
\hline 8. & Adonitol & - & 32. & D-cellobiose & - \\
\hline 9. & Beta-n-acetyl-glucosaminidase & - & 33. & Gamma-Glutamyl-Glucosaminidase & + \\
\hline 10. & D-maltose & + & 34. & Beta-xylosidae & - \\
\hline 11. & Lipase & - & 35. & Urease & - \\
\hline 12. & D-tagatose & - & 36. & Malonate & - \\
\hline 13. & alpha-glucosidase & - & 37. & Alpha-galactosidase & - \\
\hline 14. & Ornithine decarboxylase & - & 38. & Coumarate & + \\
\hline 15. & Glu-Gly-Arg-Arylamidase & - & 39. & L-lactate assimilation & - \\
\hline 16. & L-pyrrolidonyl-arylamidase & - & 40. & Beta-galactosidase & - \\
\hline 17. & Glutamyl arylamidase pNA & - & 41. & Fermentation/glucose & + \\
\hline 18. & D-Mannitol & + & 42. & Beta-alanine arylamidase pNA & - \\
\hline 19. & Palatinose & - & 43. & D-sorbitol & + \\
\hline 20. & D-TREHALOSE & + & 44. & 5-Keto-D-Gluconate & - \\
\hline 21. & Succinate alkalinization & - & 45. & Phosphate & + \\
\hline 22. & Lysine decarboxylase & + & 46. & Beta-Glucuronidase & - \\
\hline 23. & L-malate assimilation & - & 47. & 2,4-Diamino-6,7-Diisopropylpteridine resistance & + \\
\hline 24. & L-arabitol & - & & & \\
\hline
\end{tabular}

Table-2: Antibiotics susceptibility pattern against Salmonella Typhi isolates.

\begin{tabular}{|c|c|c|c|c|}
\hline \multirow[t]{2}{*}{ Classes of antibiotics } & \multirow[t]{2}{*}{ Antibiotics } & \multicolumn{2}{|c|}{ Salmonella Typhi $(n=29)$} & \multirow[t]{2}{*}{ MIC } \\
\hline & & Sensitive (\%) & Resistance (\%) & \\
\hline \multirow[t]{4}{*}{ Penicillins } & Ticarcillin & & $29(100 \%)$ & $\geq 128$ \\
\hline & Ticarcillin/clavulanic acid & $2(6.9 \%)$ & $27(93 \%)$ & 64 \\
\hline & Piperacillin & & $29(100 \%)$ & $\geq 128$ \\
\hline & Piperacillin/tazobactam & $28(96.5 \%)$ & $1(3.4 \%)$ & $\leq 4$ \\
\hline Cephalosporins $4^{\text {th }}$ generation & Cefepime & & $29(100 \%)$ & $\geq 64$ \\
\hline Cephalosporins $3^{\text {rd }}$ generation & Ceftazidime & $1(3.4 \%)$ & $28(96.5 \%)$ & $\geq 64$ \\
\hline Fluoroquinolone & Ciprofloxacin & $1(3.4 \%)$ & $28(96.5 \%)$ & 0.5 \\
\hline Monobactam & Aztreonam & & $29(100 \%)$ & $\geq 64$ \\
\hline \multirow[t]{3}{*}{ Aminoglycosides } & Amikacin & $4(13.7 \%)$ & $25(86.2 \%)$ & $\leq 2$ \\
\hline & Gentamicin & $1(3.4 \%)$ & $28(96.5 \%)$ & $\leq 1$ \\
\hline & Tobramycin & $2(6.9 \%)$ & $27(93 \%)$ & $\leq 1$ \\
\hline Sulfonamides & Trimethoprim/sulfamethoxazole & $25(86.2 \%)$ & $4(13.7 \%)$ & $\leq 20$ \\
\hline Tetracycline & Minocycline & $29(100 \%)$ & & $\leq 1$ \\
\hline \multirow[t]{2}{*}{ Carbapenems } & Imipenem & $29(100 \%)$ & & $\leq 0.25$ \\
\hline & Meropenem & $29(100 \%)$ & & $\leq 0.25$ \\
\hline
\end{tabular}

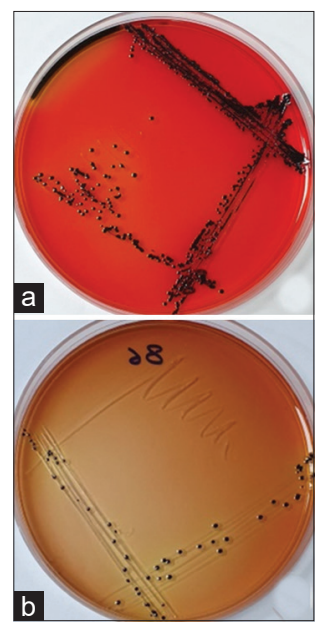

Figure-1: Representative culture media for Salmonella Typhi strains on: (a) XLD agar; (b) SS agar. monobactam (aztreonam). However, a high resistance rate was recorded against other antibiotics. Of the resistant antibiotics, ticarcillin and piperacillin were demonstrated the highest resistance (MIC $\geq 128$ ). All the strains of $S$. Typhi showed susceptibility against carbapenems (imipenem and meropenem) and tetracycline (minocycline). The data revealed that the strains of $S$. Typhi showed MDR.

\section{Discussion}

Recently, a massive number of research reports have been done to approve different tools for the identification of Salmonella spp. As the world is fighting antibiotic resistance, completing this goal requires a correct, quick, and early diagnosis. Febrile diseases like typhoid fever are among the most prevalent 
infections in Iraq. However, limited reports for determining the antibacterial susceptibility testing in Iraq were published. Therefore, this project was initiated to detect clinical strains of $S$. Typhi and determine their antibiotics susceptibility pattern.

On SS agar and XLD agar, black color colonies were considered as suspected colonies of $S$. Typhi, due to the production of $\mathrm{H}_{2} \mathrm{~S}$ (Figure-1). However, other organisms such as Proteus spp. and Citrobacter spp. may produce $\mathrm{H}_{2} \mathrm{~S}$, which forms black color colonies on XLD [18]. Hence, the identification based on culture medium is not accurate, which is in agreement with the previous report [19].

Among the sample size, 27 isolates were identified as $S$. Typhi based on biochemical tests (Table-1). The identification based on biochemical tests using Vitek 2 compact was well recommended for Gram-negative bacteria due to its accuracy [20,21]. Furthermore, 29 isolates were confirmed as $S$. Typhi based on RT-PCR. (Figure-2). The identification with Vitek 2 compact exhibited a high level of agreement with the results of RT-PCR. The same level of accuracy was observed in the previous reports $[20,22]$. In a recent study, the identification of $S$. enterica serovar Gaminara by RT-PCR was considered rapid and less expensive compared with conventional methods [23]. The current data are in accordance with a report presented by Gand et al. [24], stating that RT-PCR gives accurate detection of $S$. enterica and saves time with high efficiency, making it an ideal method of diagnosis.

The present study revealed MDR against $S$. Typhi isolated from patients in Baghdad, Iraq (Table-2). These findings are consistent with what was reported earlier in Kenya, where $68 \%$ of $S$. Typhi were MDR [25]. However, this contrasts with a study conducted in India that reported a small proportion of $S$. Typhi as MDR [26]. Whereas in Nepal, no MDR has been noted [27]. The high prevalence of MDR is perhaps because of the extensive use of antibiotics without consulting doctors, which is very common in developing countries [28], particularly in Iraq.

Among the resistant antibiotics, $100 \%$ of $S$. Typhi strains were resistant to penicillins (ticarcillin and piperacillin) which displayed the highest frequency of resistance (MIC $\geq 128$ ) (Table-2). These two antibiotics are broad-spectrum beta-lactam. Similar outcomes were found in Salmonella spp., isolated from poultry $[29,30]$. Different studies reported the resistance of Salmonella spp. to ticarcillin [31,32]. The current result is contrary to a case report conducted by Perera et al. [33] who showed a sensitivity of $S$. Typhi against piperacillin.

Piperacillin is regularly used in combination with tazobactam (piperacillin/tazobactam), which improves piperacillin efficiency by inhibiting the enzymes of beta-lactamases in different species. The present study revealed that $96.5 \%$ of $S$. Typhi strains were sensitive to piperacillin/tazobactam $(\mathrm{MIC} \leq 4)$ (Table-2). This finding is similar to the results of
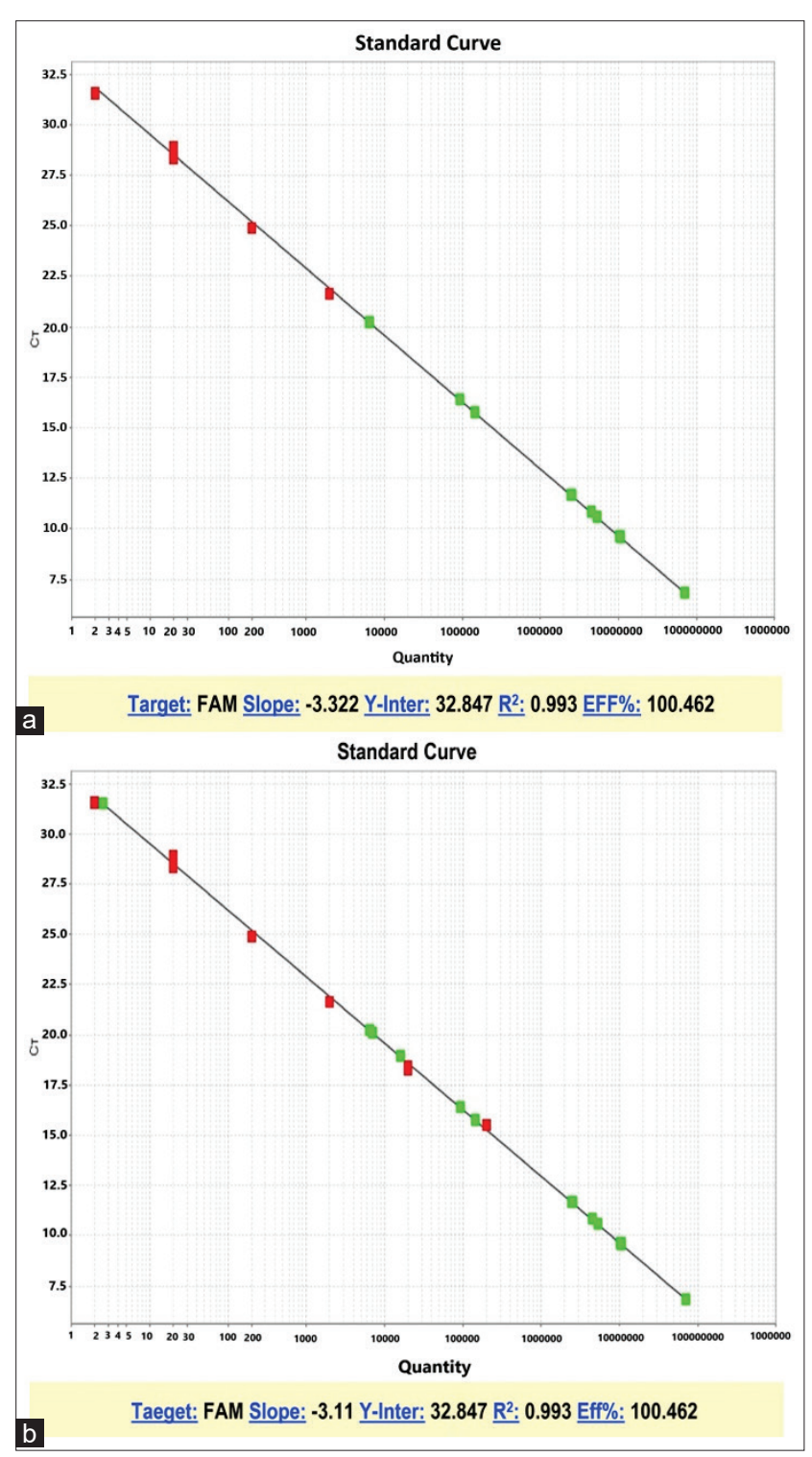

Figure-2: ( $a$ and $b$ ) The representative standard curve of real-time polymerase chain reaction of Salmonella Typhi strains.

Perera et al. [33]. Moreover, Lowman stated that piperacillin/tazobactam remains a valuable antibiotic against Gram-negative bacteria [34]. Ticarcillin is widely used in combination with clavulanic acid (ticarcillin/clavulanic acid) to treat Gram-negative bacteria [32]. However, 93\% of $S$. Typhi strains showed less resistance to ticarcillin/clavulanic acid (MIC 64). This contradicts the findings reported earlier, which demonstrated ticarcillin/clavulanic acid activity against different Gram-negative bacteria [35].

Among the isolated strains, $96.5-100 \%$ of $S$. Typhi strains displayed moderate resistance (MIC $\geq 64$ ) to cephalosporins $3^{\text {rd }} \mathrm{G}$ (ceftazidime), cephalosporins $4^{\text {th }} \mathrm{G}$ (cefepime), and monobactam (aztreonam) (Table-2). This is not in accordance with the study of Yang et al. [36] that reported the sensitivity of Salmonella spp. to ceftazidime and cefepime. The present study is comparable to the study reported earlier, indicating the resistance of $S$. enterica serotype Typhimurium to aztreonam [37]. Recent studies 
reported that the combination of aztreonam and ceftazidime-avibactam was improved the medication against Gram-negative bacteria [38-40].

Amikacin, tobramycin, and gentamicin belong to a group of antibiotics called aminoglycosides. In the present investigation, 86.2-96.5\% of $S$. Typhi strains were resistant to all antibiotics of aminoglycosides (Table-2). Lo et al. [41] reported the low activity of aminoglycosides against intracellular $S$. enterica serovar Typhimurium. Furthermore, 86.2\% of $S$. Typhi strains were found to be resistant to amikacin (Table-2). Studies conducted in India and Ethiopia demonstrated the susceptibility of $S$. Typhi to amikacin $[42,43]$, which is not in agreement with our results. $S$. Typhi strains $(96.5 \%)$ were found to be resistant to gentamicin (MIC $\leq 1)$ (Table-2), which is not in accordance with previous reports that showed a susceptibility of $S$. Typhi to gentamicin [25,43]. Moreover, recent data reported that Gram-negative bacteria were sensitive to gentamicin [40]. The present study specified the resistance of $93 \%$ of $S$. Typhi strains to tobramycin (Table-2). This is strongly contradicted with a study conducted in Baghdad, Iraq, which demonstrated the sensitivity of $80 \%$ of $S$. Typhi strains to tobramycin [44]. This is a clear indication of the resistance increasing of $S$. Typhi to tobramycin.

Moreover, $96.5 \%$ of strains of $S$. Typhi have also exhibited resistance to ciprofloxacin (Table-2). The previous studies reported increasing numbers of resistance of $S$. Typhi to ciprofloxacin $[25,45,46]$. However, another study in Ethiopia revealed the susceptibility of $S$. Typhi to ciprofloxacin [43], which is contrary to the current research. As per the literature review, ciprofloxacin is a substitute antibiotic for the treatment of MDR-Salmonella spp. Reduced ciprofloxacin sensitivity has a significant risk to the failure of typhoid fever medication, particularly in developing countries. Among the Middle East countries, Iraq was shown the highest sensitivity reduction in ciprofloxacin [10], which is in accordance with the present study. Moreover, the current study is comparable to a study from Baghdad, where $S$. Typhi was resistant to ciprofloxacin [44].

On the other hand, the study documented the sensitivity of $S$. Typhi against imipenem, meropenem, minocycline, and trimethoprim/sulfamethoxazole (Table-2). In contrast, a study in Indonesia reported resistance of $S$. Typhi to imipenem, meropenem, and trimethoprim/sulfamethoxazole [47], while a study in Nepal displayed a sensitivity of $S$. Typhi to trimetho$\mathrm{prim} / \mathrm{sulfamethoxazole} \mathrm{[27].} \mathrm{Whereas} \mathrm{in} \mathrm{Pakistan,} \mathrm{a}$ high resistance rate of $S$. Typhi to trimethoprim/sulfamethoxazole was observed [48]. Minocycline has been an effective anti-MDR antibacterial for over the past 30 years [49]. In contrast to our results, a study from South Korea reported resistance of 30 strains of Salmonella spp. to minocycline [50].

According to the present findings, $S$. Typhi strains were shown the highest resistance against ticarcillin, piperacillin, and cefepime. Therefore, we strongly advise not to use these antibiotics for typhoid treatment. On the other hand, imipenem and meropenem were presented the highest sensitivity.

\section{Conclusion}

The study concluded that the use of Vitek 2 compact and RT-PCR in detecting $S$. Typhi is giving accurate results which are very important for the diagnosis of typhoid. According to the above findings, we observed increasing numbers of antibiotic-resistant $S$. Typhi, which is required to raise the level of awareness of consuming antibiotics. Continuous surveillance of the antibacterial sensitivity testing and a reasonable dosage of antibiotics should be maintained to avoid the outbreak of multidrug resistance.

\section{Authors' Contributions}

HAS: Wrote and corrected the manuscript as well as analyzed the data. HAS, AMA, and MNF: Did the laboratory work, including Vitek 2 compact and realtime PCR. ZMR: Collected the samples and managed the data as well as laboratory work such as Vitek 2 compact antibiotics susceptibility testing. All authors have read and approved the final manuscript.

\section{Acknowledgments}

We are thankful to Al-Nokhba Diagnostic Centre for providing all the facilities required to accomplish this study. The authors did not receive any funds for this study.

\section{Competing Interests}

The authors declare that they have no competing interests.

\section{Publisher's Note}

Veterinary World remains neutral with regard to jurisdictional claims in published institutional affiliation.

\section{References}

1. Lamas, A., Miranda, J.M., Regal, P., Vázquez, B., Franco, C.M. and Cepeda, A. (2018) A comprehensive review of non-enterica subspecies of Salmonella enterica. Microbiol. Res., 206: 60-73.

2. Gal-Mor, O., Boyle, E.C. and Grassl, G.A. (2014) Same species, different diseases: How and why typhoidal and non-typhoidal Salmonella enterica serovars differ. Front. Microbiol., 5: 391-391.

3. Pouzol, S., Tanmoy, A.M., Ahmed, D., Khanam, F., Brooks, W.A., Bhuyan, G.S., Fabre, L., Bryant, J.E., Gustin, M.P., Vanhems, P., Carman, B., Weill, F.X., Qadri, F., Saha, S. and Endtz, H. (2019) Clinical evaluation of a multiplex PCR for the detection of Salmonella enterica Serovars Typhi and Paratyphi A from blood specimens in a high-endemic setting. Am. J. Trop. Med. Hyg., 101(3): 513-520.

4. Alzwghaibi, A.B., Yahyaraeyat, R., Fasaei, B.N., Langeroudi, A.G. and Salehi, T.Z. (2018) Rapid molecular identification and differentiation of common Salmonella serovars isolated from poultry, domestic animals and foodstuff using multiplex PCR assay. Arch. Microbiol., 200(7): 1009-1016.

5. Igbinosa, I.H., Beshiru, A., Ikediashi, S.C. and Igbinosa, E.O. (2020) Identification and characterization of 
Salmonella serovars isolated from pig farms in Benin city, Edo State, Nigeria: One health perspective. Microb. Drug Resist., 27(2): 258-267.

6. Mogasale, V., Maskery, B., Ochiai, R.L., Lee, J.S., Mogasale, V.V., Ramani, E., Kim, Y.E., Park, J.K. and Wierzba, T.F. (2014) Burden of typhoid fever in low-income and middle-income countries: A systematic, literature-based update with risk-factor adjustment. Lancet Glob. Health, 2(10): e570-e580.

7. Korzeniewski, K. (2006) The epidemiological situation in Iraq. Przegl. Epidemiol., 60(4): 845-55.

8. Doyle, M.P., Loneragan, G.H., Scott, H.M. and Singer, R.S. (2013) Antimicrobial resistance: Challenges and perspectives. Compr. Rev. Food Sci. Food Saf., 12: 234-248.

9. Klemm, E.J., Shakoor, S., Page, A.J., Qamar, F.N., Judge, K., Saeed, D.K., Wong, V.K., Dallman, T.J., Nair, S., Baker, S., Shaheen, G., Qureshi, S., Yousafzai, M.T., Saleem, M.K., Hasan, Z., Dougan, G. and Hasan, R. (2018) Emergence of an extensively drug-resistant Salmonella enterica Serovar Typhi clone harboring a promiscuous plasmid encoding resistance to fluoroquinolones and third-generation cephalosporins. mBio, 9(1): e00105-18.

10. Rahman, B.A., Wasfy, M.O., Maksoud, M.A., Hanna, N., Dueger, E. and House, B. (2014) Multi-drug resistance and reduced susceptibility to ciprofloxacin among Salmonella enterica serovar Typhi isolates from the Middle East and Central Asia. New Microbes New Infect., 2(4): 88-92.

11. Fàbrega, A. and Vila, J. (2013) Salmonella enterica Serovar Typhimurium skills to succeed in the host: Virulence and regulation. Clin. Microbiol. Rev., 26(2): 308-341.

12. Wain, J., Diep, T.S., Bay, P.V., Walsh, A.L., Vinh, H., Duong, N.M., Ho, V.A., Hien, T.T., Farrar, J., White, N.J., Parry, C.M. and Day, N.P. (2008) Specimens and culture media for the laboratory diagnosis of typhoid fever. $J$. Infect. Dev. Ctries., 2(6): 469-474.

13. Song, J.H., Cho, H., Park, M.Y., Na, D.S., Moon, H.B. and Pai, C.H. (1993) Detection of Salmonella Typhi in the blood of patients with typhoid fever by polymerase chain reaction. J. Clin. Microbiol., 31(6): 1439-1443.

14. Hashimoto, Y., Itho, Y., Fujinaga, Y., Khan, A.Q., Sultana, F., Miyake, M., Hirose, K., Yamamoto, H. and Ezaki, T. (1995) Development of nested PCR based on the ViaB sequence to detect Salmonella Typhi. J. Clin. Microbiol., 33(3): 775-777.

15. Salman, H.A., Senthilkumar, R., Imran, K. and Selvam, K.P. (2017) Isolation and Typing of Streptococcus mutans and Streptococcus sobrinus from caries-active subjects. Contemp. Clin. Dent., 8(4): 587-593.

16. Salman, H.A. and Senthilkumar, R.J.B. (2020) Genotypic variations of mutans streptococci isolated from dental caries by REP-PCR. Baghdad Sci. J., 17(4): 1133-1133.

17. Alanazi, M.Q., Alqahtani, F.Y. and Aleanizy, F.S. (2018) An evaluation of $E$. coli in urinary tract infection in emergency department at KAMC in Riyadh, Saudi Arabia: Retrospective study. Ann. Clin. Microbiol. Antimicrob., 17(1): 3 .

18. Rambach, A. (1990) New plate medium for facilitated differentiation of Salmonella spp. from Proteus spp. and other enteric bacteria. Appl. Environ. Microbiol., 56(1): 301-303.

19. Park, S.H., Ryu, S. and Kang, D.H. (2012) Development of an improved selective and differential medium for isolation of Salmonella spp. J. Clin. Microbiol., 50(10): 3222-3226.

20. Monteiro, A.C.M., Fortaleza, C.M.C., Ferreira, A.M., Cavalcante, R.D.S., Mondelli, A.L., Bagagli, E. and da Cunha, M.D.L. (2016) Comparison of methods for the identification of microorganisms isolated from blood cultures. Ann. Clin. Microbiol. Antimicrob., 15(1): 45-45.

21. Maina, D. and Kagotho, E. (2014) Suitability of vitek 2 system in identification and susceptibility testing of gram-negative bacteremias by direct inoculation. East Afr. Med. J., 91(4): 115-118.

22. Nakasone, I., Kinjo, T., Yamane, N., Kisanuki, K. and
Shiohira, C.M. (2007) Laboratory-based evaluation of the colorimetric VITEK-2 compact system for species identification and of the advanced expert system for detection of antimicrobial resistances: VITEK-2 compact system identification and antimicrobial susceptibility testing. Diagn. Microbiol. Infect. Dis., 58(2): 191-198.

23. Kasturi, K.N. (2020) A real-time PCR for rapid identification of Salmonella enterica Gaminara serovar. J. Microbiol. Methods, 169: 105729.

24. Gand, M., Mattheus, W., Saltykova, A., Roosens, N., Dierick, K., Marchal, K., De Keersmaecker, S.C.J. and Bertrand, S. (2019) Development of a real-time PCR method for the genoserotyping of Salmonella Paratyphi B variant Java. Appl. Microbiol. Biotechnol., 103(12): 4987-4996.

25. Mutai, W.C., Muigai, A.W.T., Waiyaki, P. and Kariuki, S. (2018) Multi-drug resistant Salmonella enterica serovar Typhi isolates with reduced susceptibility to ciprofloxacin in Kenya. BMC Microbiol., 18(1): 187.

26. Shetty, A.K., Shetty, I.N., Furtado, Z.V., Antony, B. and Boloor, R. (2012) Antibiogram of Salmonella isolates from blood with an emphasis on nalidixic Acid and chloramphenicol susceptibility in a tertiary care hospital in coastal Karnataka: A prospective study. J. Lab. Physicians, 4(2): 74-77.

27. Chand, H.J., Rijal, K.R., Neupane, B., Sharma, V.K. and Jha, B. (2014) Re-emergence of susceptibility to conventional first line drugs in Salmonella isolates from enteric fever patients in Nepal. J. Infect. Dev. Ctries., 8(11): 1483-1487.

28. Omulo, S., Thumbi, S.M., Njenga, M.K. and Call, D.R. (2015) A review of 40 years of enteric antimicrobial resistance research in Eastern Africa: What can be done better? Antimicrob. Resist. Infect. Control, 4(1): 1-1.

29. Mir, I.A., Kashyap, S.K. and Maherchandani, S. (2015) Isolation, serotype diversity and antibiogram of Salmonella enterica isolated from different species of poultry in India. Asian Pac. J. Trop. Biomed., 5(7): 561-567.

30. Mezali, L. and Hamdi, T.M. (2012) Prevalence and antimicrobial resistance of Salmonella isolated from meat and meat products in Algiers (Algeria). Foodborne Pathog. Dis., 9(6): 522-529.

31. Johnson, J.M., Rajic, A. and McMullen, L.M. (2005) Antimicrobial resistance of selected Salmonella isolates from food animals and food in Alberta. Can. Vet. J., 46(2): 141-146.

32. Reed, M.D. (1998) Rational prescribing of extended-spectrum penicillin beta-lactamase inhibitor combinations: Focus on ticarcillin/clavulanic acid. Ann. Pharmacother., 32(1): S17-S21.

33. Perera, N., Geary, C., Wiselka, M., Rajakumar, K. and Swann, R.A. (2007) Mixed Salmonella infection: Case report and review of the literature. J. Travel Med., 14(2): 134-135.

34. Lowman, W. (2013) Re-evaluating the role of piperacillin-tazobactam in the treatment of hospital-acquired infections: Original research. Southern Afr. J. Epidemiol. Infect., 28(1): 16-21.

35. Rouhan, D., Le Noc, P. and Robert, J. (1985) Effect of a ticarcillin-clavulanic acid (timentin) combination on bacteria resistant to ticarcillin. Pathol. Biol., 33(5 Pt 2): 461-465.

36. Yang, C., Li, H., Zhang, T., Chu, Y., Zuo, J. and Chen, D. (2020) Study on antibiotic susceptibility of Salmonella Typhimurium L forms to the third and fourth generation cephalosporins. Sci. Rep., 10: 3042.

37. Kariuki, S., Okoro, C., Kiiru, J., Njoroge, S., Omuse, G., Langridge, G., Kingsley, R.A., Dougan, G. and Revathi, G. (2015) Ceftriaxone-resistant Salmonella enterica serotype Typhimurium sequence type 313 from Kenyan patients is associated with the blaCTX-M-15 gene on a novel IncHI2 plasmid. Antimicrob. Agents Chemother., 59(6): 3133-3139.

38. Jayol, A., Nordmann, P., Poirel, L. and Dubois, V. (2018) Ceftazidime/avibactam alone or in combination with 
aztreonam against colistin-resistant and carbapenemase-producing Klebsiella pneumoniae. J. Antimicrob. Chemother., 73(2): 542-544.

39. Wenzler, E., Deraedt, M.F., Harrington, A.T. and Danizger, L.H. (2017) Synergistic activity of ceftazidime-avibactam and aztreonam against serine and metallo- $\beta$-lactamase-producing gram-negative pathogens. Diagn. Microbiol. Infect. Dis., 88(4): 352-354.

40. Emeraud, C., Escaut, L., Boucly, A., Fortineau, N., Bonnin, R.A., Naas, T. and Dortet, L. (2019) Aztreonam plus clavulanate, tazobactam, or avibactam for treatment of Infections caused by metallo- $\beta$-lactamase-producing gram-negative bacteria. Antimicrob. Agents Chemother., 63(5): e00010-19.

41. Lo, J.H., Kulp, S.K., Chen, C.S. and Chiu, H.C. (2014) Sensitization of intracellular Salmonella enterica serovar Typhimurium to aminoglycosides in vitro and in vivo by a host-targeted antimicrobial agent. Antimicrob. Agents Chemother., 58(12): 7375-7382.

42. Mandal, S., Debmandal, M. and Pal, N.K. (2012) Antibiotic resistance of Salmonella enterica serovar Typhi in Kolkata, India, and in vitro experiments on effect of combined chemotherapy. Sci. World J., 2012: 454059.

43. Tosisa, W., Mihret, A., Ararsa, A., Eguale, T. and Abebe, T. (2020) Prevalence and antimicrobial susceptibility of Salmonella and Shigella species isolated from diarrheic children in Ambo town. BMC Pediatr., 20(1): 91.

44. Rasool, K.H., Hussein, N.H. and Taha, B.M. (2020) Molecular detection of gyrA gene in Salmonella enterica serovar Typhi isolated from typhoid patients in Baghdad. Pak. J. Biolog. Sci., 23(10): 1303-1309.

45. Lutterloh, E., Likaka, A., Sejvar, J., Manda, R., Naiene, J., Monroe, S.S., Khaila, T., Chilima, B., Mallewa, M.,
Kampondeni, S.D., Lowther, S.A., Capewell, L., Date, K., Townes, D., Redwood, Y., Schier, J.G., Nygren, B., Barr, B.T., Demby, A., Phiri, A., Lungu, R., Kaphiyo, J., Humphrys, M., Talkington, D., Joyce, K., Stockman, L.J., Armstrong, G.L. and Mintz, E. (2012) Multidrug-resistant typhoid fever with neurologic findings on the MalawiMozambique border. Clin. Infect. Dis., 54(8): 1100-1106.

46. Date, K.A., Newton, A.E., Medalla, F., Blackstock, A., Richardson, L., McCullough, A., Mintz, E.D. and Mahon, B.E. (2016) Changing patterns in enteric fever incidence and increasing antibiotic resistance of enteric fever isolates in the United States, 2008-2012. Clin. Infect. Dis., 63(3): 322-329.

47. Lugito, N.P.H. and Cucunawangsih. (2017) Antimicrobial resistance of Salmonella enterica serovars Typhi and Paratyphi isolates from a general hospital in Karawaci, Tangerang, Indonesia: A five-year review. Int. J. Microbiol., 2017: 6215136.

48. Qamar, F.N., Azmatullah, A., Kazi, A.M., Khan, E. and Zaidi, A.K. (2014) A three-year review of antimicrobial resistance of Salmonella enterica serovars Typhi and Paratyphi A in Pakistan. J. Infect. Dev. Ctries., 8(8): 981-986.

49. Asadi, A., Abdi, M., Kouhsari, E., Panahi, P., Sholeh, M., Sadeghifard, N., Amiriani, T., Ahmadi, A., Maleki, A. and Gholami, M. (2020) Minocycline, focus on mechanisms of resistance, antibacterial activity, and clinical effectiveness: Back to the future. J. Glob. Antimicrob. Resist., 22: 161-174.

50. Cha, S.Y., Kang, M., Yoon, R.H., Park, C.K., Moon, O.K. and Jang, H.K. (2013) Prevalence and antimicrobial susceptibility of Salmonella isolates in Pekin ducks from South Korea. Comp. Immunol. Microbiol. Infect. Dis., 36(5): 473-479. 